\section{Selective Response to Omalizumab in a Patient With Concomitant ncMCAS and POTS: What Does it Teach us About the Underlying Disease?}

\author{
Kacar M, Denman S, Savic S \\ Department of Clinical Immunology and Allergy, St James's \\ University Hospital, Beckett Street, Leeds, UK
}

J Investig Allergol Clin Immunol 2018; Vol. 28(4): 261-263 doi: 10.18176/jiaci.0251

Key words: Nonclonal mast cell activation syndrome. Postural orthostatic tachycardia syndrome. Omalizumab.

Palabras clave: Síndrome de activación mastocitaria no clonal. Síndrome de taquicardia postural ortostática. Omalizumab.

Nonclonal mast cell activation syndrome (ncMCAS) is a disorder characterized by episodic mast cell degranulation and the effect of this process on target organ systems [1]. To meet the diagnostic criteria for ncMCAS, the episodes must involve 2 or more organ systems in the absence of a primary or secondary cause of mast cell activation (MCA), including systemic mastocytosis. Furthermore, there must be a recorded rise in urine or serum levels of mast cell degranulation markers, and the condition must be responsive to antimediator therapy such as histamine 1 and 2 receptor antagonists, leukotriene antagonists, and mast cell stabilizers [1]. A proportion of patients with ncMCAS also have concomitant postural orthostatic tachycardia syndrome (POTS) [2]. POTS is a heterogenous form of orthostatic intolerance characterized by an increase in the heart rate of at least $30 \mathrm{bpm}$ within 10 minutes of assuming an upright posture. POTS commonly presents with cardiac symptoms (eg, palpitations, lightheadedness, and dyspnea) and noncardiac symptoms (eg, headache, exercise intolerance, fatigue) [3]. The link between these 2 clinical entities is not entirely clear, although it has been suggested that the vasodilator effect of MCA-related mediators such as histamine, norepinephrine, and neuropeptide Y (NPY) results in increased sympathetic activity, which in turn leads to orthostatic tachycardia [2]. In recent years, a handful of patients with recalcitrant ncMCAS were successfully treated with omalizumab, the monoclonal antibody to $\operatorname{IgE}$ [4].

Here, we present a patient with coexisting ncMCAS and POTS, whose ncMCAS-related symptoms responded fully to omalizumab but who remains extremely symptomatic with POTS.

This 21-year-old British male initially presented at the age of 10 years with recurrent episodes of unexplained anaphylaxis occurring on a 2 -weekly to 2 -monthly basis. There were no obvious triggers to these episodes. In addition to anaphylaxis, he reported having more persistent symptoms, including almost daily flushing, profuse sweating, hives, and occasional wheeziness. In his late teens he developed episodic diarrhea, erythromelalgia, severe dizziness associated with hypotension, and tachycardia and experienced loss of consciousness.
Investigations at the time showed no evidence of an allergic disorder. However, serum mast cell tryptase levels were periodically elevated, ranging from $10 \mathrm{mg} / \mathrm{mL}$ to $20 \mathrm{ng} / \mathrm{mL}$ between attacks, but increasing to $>100 \mathrm{ng} / \mathrm{mL}$ following episodes of anaphylaxis. Subsequent investigations to exclude a clonal mast cell disorder were performed in another center. This included a complete bone marrow study to assess the number of mast cells and typical histological lesions of systemic mastocytosis, such as multifocal dense, sharply demarcated mast cell infiltrates [5]. Furthermore, bone marrow aspirate was examined for the number of mast cells and their morphological features, and immunophenotyping studies were performed to assess expression of CD2 and CD25, which are typically found on mast cells from patients with systemic mastocytosis [5]. Lastly, cKIT mutation analysis yielded negative results; however, we do not know if this was performed from purified mast cells, as currently recommended [6]. Altogether, these investigations showed no evidence of clonal mast cell proliferation.

Blood tests including measurements of plasma levels of epinephrine, aldosterone, rennin, 5-hydroxyindoleacetic acid (5-HIAA), and gut hormones were all normal. An extensive autoantibody screen for POTS-related autoantibodies was negative. This included anti- $\alpha-1$ adrenergic, anti- $\alpha-2$ adrenergic, anti- $\beta-1$ adrenergic, and anti- $\beta-2$ adrenergic receptor antibodies, as well as antimuscarinic cholinergic receptor 1, 2, 3, 4, and 5 antibodies. The patient and close family members (mother, father, and a brother) underwent further genetic tests. Although there was no family history of ncMCAS/POTS, the severity and early onset of the patient's illness led us to consider a monogenic cause of this rare clinical phenotype. However, whole exome sequencing carried out on the patient's DNA failed to reveal an obvious genetic cause.

The patient received numerous treatments for ncMCASrelated symptoms, including high-dose $\mathrm{H}_{1}$ nonsedating antihistamines, $\mathrm{H}_{2}$ antihistamines, montelukast, sodium cromoglycate, and the tyrosine kinase receptor inhibitor imatinib, which is an effective inhibitor of wild-type KIT; however, it is currently indicated for treatment of systemic mastocytosis with imatinib-sensitive KIT mutations only [7]. Considering that imatinib might cause potentially dangerous adverse effects, it is not recommended for routine use in ncMCAS. In this case, the permission to use imatinib was obtained from the hospital's Drugs and Therapeutics Committee, and appropriate consent was obtained from the patient prior to commencing the treatment. Unfortunately, none of the medications were fully effective, resulting in only partial improvement of the patient's condition. He also tried several medications to control his POTS-related symptoms. These included moxonidine and amlodipine for hypertension, pyridostigmine for orthostatic hypotension, pregabalin for erythromelalgia, and propantheline for hyperhidrosis. These drugs were discontinued owing to lack of effect (moxonidine, amlodipine, pregabalin) or intolerable adverse effects (propantheline, pyridostigmine).

Attempts were made to treat POTS-related symptoms, assuming an autoimmune basis for the condition. The patient failed to respond to either therapeutic plasmapheresis or highdose, immunomodulatory intravenous immunoglobulin. 
Subcutaneous omalizumab was initially commenced at a dose of $300 \mathrm{mg}$ every 4 weeks, but the frequency of injections later increased to 3-weekly owing to mild breakthrough MCAS symptoms. Since commencing omalizumab 2 years ago, the patient has had no further episodes of urticaria, anaphylaxis, or loss of consciousness. However, he remains severely affected by POTS-associated symptoms including diarrhea, excessive sweating, and erythromelalgia.

The co-occurrence of MCAS and POTS in some patients suggests that both clinical entities might share a common pathological pathway. Indeed, mast cells might contain chemical mediators other than histamine, with physiological effects that could reproduce POTS-related symptoms [2]. It might therefore be expected that agents preventing mast cell degranulation, such as omalizumab, could be effective for both disorders. The effect of omalizumab on non-IgE-mediated MCA is now believed to be exerted via 2 mechanisms: first, binding to specific IgE molecules that recognize autoantigens and block their function [8]; and second, down-regulation of the mast cell high-affinity IgE receptor, FCERI [4]. Failure of omalizumab in this case to alleviate POTS symptoms might be due to a differential mast cell activation pathway not disrupted by the anti-IgE drug. Differential mast cell activation without degranulation has previously been observed in the context of various inflammatory processes, and may be triggered by numerous mediators such as cytokines, hormones, and growth factors, as well as microbial antigens [9].

Another feature common to these conditions is their likely autoimmune etiology. Recent studies have pointed to an autoimmune etiology of chronic spontaneous urticaria through the action of $\operatorname{IgE}$ isotype antibodies to thyroid peroxidase which cross-react with the high-affinity mast cell IgE receptor FCERI [10]. A similar mechanism has been thought to be responsible in ncMCAS. In articles describing the association of autoimmunity and POTS, the causative autoantibodies were of the IgG isotype [11]. The initial failure of omalizumab to neutralize potential POTScausing IgE antibodies could therefore be attributed to its specificity towards the wrong immunoglobulin isotype. The subsequent failure of immunomodulatory intravenous immunoglobulin and plasmapheresis in eliciting clinical improvement, however, could be attributed to their inability to accurately target the casuative antibodies, or to absence of the autoantibodies themselves. Case reports describing treatment of POTS symptoms with plasmapheresis [12] or high-dose immunoglobulin [13] involved proven anti-NMDA receptor autoantibodies, which were not found in this patient's case.

A positive therapeutic response to a targeted treatment can occasionally be extremely helpful to delineate an underlying pathological mechanism. The introduction of omalizumab for treatment of chronic spontaneous urticaria has certainly helped to improve our understanding of this condition. However, in this case, the response to omalizumab was only partial, suggesting that 2 clinical entities, ncMCAS and POTS, while occurring concurrently and in the same patient, might not be caused by entirely identical pathological processes. Nevertheless, symptoms of nsMCAS and POTS can be triggered by mast cell mediators. In addition, both conditions often have an autoimmune basis. Therefore, it is likely that a common pathological process, at least in part and yet to be fully delineated, underlies these 2 conditions.

\section{Acknowledgement}

We would like to thank the patient and his family for graciously allowing us to share our findings and for their constant willingness to provide detailed information.

\section{Funding}

The authors declare that no funding was received for the present study.

\section{Conflicts of Interest}

SS and SD received honoraria from Novartis for advisory board participation and speaking at educational meetings. MK has no conflicts of interest to declare.

\section{References}

1. Valent $P$, Akin $C$, Arock M, Brockow K, Butterfield JH, Carter $M C$, et al. Definitions, criteria and global classification of mast cell disorders with special reference to mast cell activation syndromes: A consensus proposal. Int Arch Allergy Immunol. 2012; 157:215-25.

2. Shibao C, Arzubiaga C, Roberts LJ, Raj S, Black B, Harris P, et al. Hyperadrenergic postural tachycardia syndrome in mast cell activation disorders. Hypertens (Dallas, Tex 1979). 2005;45:38590. doi:10.1161/01.HYP.0000158259.68614.40.

3. Garland EM, Celedonio JE, Raj SR. Postural Tachycardia Syndrome: Beyond Orthostatic Intolerance. Curr Neurol Neurosci Rep. 2015;15.

4. Chang TW, Shiung YY. Anti-lgE as a mast cell-stabilizing therapeutic agent. J Allergy Clin Immunol. 2006;117:1203-12.

5. Valent P, Horny HP, Escribano L, Longley BJ, Li CY, Schwartz LB, et al. Diagnostic criteria and classification of mastocytosis: a consensus proposal. Leuk Res. 2001;25(7):603-25

6. Garcia-Montero AC, Jara-Acevedo M, Teodosio C, et al. KIT mutation in mast cells and other bone marrow hematopoietic cell lineages in systemic mast cell disorders: a prospective study of the Spanish Network on Mastocytosis (REMA) in a series of 113 patients. Blood. 2006;108(7):2366-72

7. Pardanani A, Tefferi A. Imatinib targets other than bcr/ abl and their clinical relevance in myeloid disorders. Blood. 2004;104(7):1931-9.

8. Maurer M, Altrichter $\mathrm{S}$, Bieber T, Biedermann T, Bräutigam M, Seyfried S, et al. Efficacy and safety of omalizumab in patients with chronic urticaria who exhibit IgE against thyroperoxidase. J Allergy Clin Immunol. 2011;128:202-9.

9. Theoharides TC, Kempuraj D, Tagen M, Conti $P$, Kalogeromitros D. Differential release of mast cell mediators and the pathogenesis of inflammation. Immunological Rev. 2007;217:65-78.

10. Altrichter S, Peter HJ, Pisarevskaja D, Metz M, Martus $P$, Maurer $M$. Ige mediated autoallergy against thyroid peroxidase - a novel pathomechanism of chronic spontaneous urticaria? PLoS ONE. 2011;6:e14794. doi:10.1371/journal. pone.0014794. 
11. Fedorowski A, Li H, Yu X, Koelsch KA, Harris VM, Liles C, et al. Antiadrenergic autoimmunity in postural tachycardia syndrome. Europace. 2017;19:1211-9.

12. Blitshteyn S, Brook J. Postural tachycardia syndrome (POTS) with anti-NMDA receptor antibodies after human papillomavirus vaccination. Immunol Res. 2016;65:1-3.

13. Gibbons $\mathrm{CH}$, Vernino $\mathrm{S}$ a, Freeman $\mathrm{R}$. Combined immunomodulatory therapy in autoimmune autonomic ganglionopathy. Arch Neurol. 2008;65:213-7. doi:10.1001/ archneurol.2007.60.

Manuscript received December 5, 2017; accepted for publication March 5, 2018

Sinisa Savic

Department of Clinical Immunology and Allergy St James University

Beckett Street Leeds, UK Email: s.savic@leeds.ac.uk 